\title{
Demystifying the bias from selective inference: A revisit to Dawid's treatment selection problem
}

\author{
Jiannan $\mathrm{Lu}^{*}$ and Alex Deng \\ Analysis and Experimentation, Microsoft Corporation
}

\begin{abstract}
We extend the heuristic discussion in Senn (2008) on the bias from selective inference for the treatment selection problem (Dawid 1994), by deriving the closed-form expression for the selection bias. We illustrate the advantages of our theoretical results through numerical and simulated examples.
\end{abstract}

Keywords: Bayesian inference; posterior mean; selection paradox; multivariate truncated normal.

\section{INTRODUCTION}

Selective inference gained popularity in recent years (e.g., Lockhart et al. 2014, G'Sell et al. 2016. Reid and Tibshirani 2016). To quote Dawid (1994), “... a great deal of statistical practice involves, explicitly or implicitly, a two stage analysis of the data. At the first stage, the data are used to identify a particular parameter on which attention is to focus; the second stage then attempts to make inferences about the selected parameter." Consequently, the results (e.g., point estimates, $p$-values) produced by selective inference are generally "cherry-picked" (Taylor and Tibshirani 2015), and therefore it is of great importance for practitioners to conduct "exact post-selection inference" (e.g., Tibshirani et al. 2014; Lee et al. 2015).

To demonstrate the importance of "exact post-selection inference," in this paper we focus on the "bias" of the posterior mean associated with the most extreme observation (formally defined later,

*Address for correspondence: Jiannan Lu, Microsoft Corporation, One Microsoft Way, Redmond, Washington 98052, USA. Email: jiannl@microsoft.com 
and henceforth referred to as "selection bias") in the treatment selection problem (Dawid 1994), which is not only fundamental in theory, but also of great practical importance in, e.g., agricultural studies, clinical trials, and large-scale online experiments (Kohavi et al. 2013). In an illuminating paper, Senn (2008) provided a heuristic explanation that the existence of selection bias depended on the prior distribution, and upheld Dawid's claim that the fact that selection bias did not exist in some standard cases was a consequence of using certain conjugate prior. In this paper, we relax the modeling assumptions in Senn (2008) and derive the closed-form expression for the selection bias. Consequently, our work can serve as a complement of the heuristic explanation provided by $\operatorname{Senn}(2008)$, and is useful from both theoretical and practical perspectives.

The paper proceeds as follows. Section 2 reviews the treatment selection problem, defines the selection bias, and describes the Bayesian inference framework which the remaining parts of the paper are based on. Section 3 derives the closed-form expression for the selection bias. Section 4 highlights numerical and simulated examples that illustrates the advantages of our theoretical results. Section 5 concludes and discusses future directions.

\section{BAYESIAN INFERENCE FOR TREATMENT SELECTION PROBLEM}

\subsection{Treatment Selection Problem and Selection Bias}

Consider an experiment with $p \geq 2$ treatment arms. For $i=1, \ldots, p$, let $\mu_{i}$ denote the mean yield of the $i$ th treatment arm. After running the experiment, we observe the sample mean yield of the $i$ th treatment arm, denoted as $X_{i}$. Let

$$
i^{*}=\underset{1 \leq i \leq p}{\arg \max } X_{i}
$$

denote the index of the largest observation. The focus of selective inference is on $\mu_{i^{*}}$, which relies on $X_{1}, \ldots, X_{p}$. We let $\mathrm{E}\left(\mu_{i^{*}} \mid X_{i^{*}}\right)$ be the posterior mean of $\mu_{i^{*}}$ as if it were selected before the experiment, and

$$
\mathrm{E}\left(\mu_{i^{*}} \mid X_{i^{*}}, X_{i^{*}}=\max X_{i}\right)
$$


be the "exact post-selection" posterior mean of $\mu_{i^{*}}$, which takes the selection into account. Following Senn (2008), we define the selection bias as

$$
\Delta=\mathrm{E}\left(\mu_{i^{*}} \mid X_{i^{*}}\right)-\mathrm{E}\left(\mu_{i^{*}} \mid X_{i^{*}}, X_{i^{*}}=\max X_{i}\right)
$$

Having defined the selection bias, we briefly discuss the "selection paradox" in Dawid (1994), i.e., "since Bayesian posterior distributions are already fully conditioned on the data, the posterior distribution of any quantity is the same, whether it was chosen in advance or selected in the light of the data." In other words, if we define the selection bias as

$$
\tilde{\Delta}=\mathrm{E}\left(\mu_{i^{*}} \mid X_{1}, \ldots, X_{p}\right)-\mathrm{E}\left(\mu_{i^{*}} \mid X_{1}, \ldots, X_{p}, X_{i^{*}}=\max X_{i}\right)
$$

then indeed $\tilde{\Delta}=0$.

\subsection{The Normal-Normal Model}

Let $\boldsymbol{\mu}=\left(\mu_{1}, \ldots, \mu_{p}\right)^{\prime}$ and $\boldsymbol{X}=\left(X_{1}, \ldots, X_{p}\right)^{\prime}$. Following Dawid (1994), we treat them as random vectors. We generalize Senn (2008) and assume that

$$
\boldsymbol{\mu} \sim N\left(\mathbf{0}, \boldsymbol{\Sigma}_{0}\right), \quad \boldsymbol{X} \mid \boldsymbol{\mu} \sim N(\boldsymbol{\mu}, \boldsymbol{\Sigma}),
$$

where

$$
\boldsymbol{\Sigma}_{0}=\gamma^{2} \boldsymbol{I}_{p}+\left(1-\gamma^{2}\right) \mathbf{1}_{p} \mathbf{1}_{p}^{\prime}, \quad \boldsymbol{\Sigma}=\sigma^{2}\left\{\eta^{2} \boldsymbol{I}_{p}+\left(1-\eta^{2}\right) \mathbf{1}_{p} \mathbf{1}_{p}^{\prime}\right\}, \quad 0 \leq \gamma, \eta \leq 1
$$

To interpret (3) we let $X_{i}=\mu_{i}+\epsilon_{i}$, where $\mu_{i}$ is generated by

$$
\phi \sim N\left(0,1-\gamma^{2}\right), \quad \mu_{i} \mid \phi \sim N\left(\phi, \gamma^{2}\right)
$$

and $\epsilon_{i}$ is generated by

$$
\xi \sim N\left\{0,\left(1-\eta^{2}\right) \sigma^{2}\right\}, \quad \epsilon_{i} \mid \xi \sim N\left(\xi, \eta^{2} \sigma^{2}\right)
$$

Note that $\eta=1$ in $\operatorname{Senn}(2008)$, and we relax this assumption by allowing correlated errors. 


\subsection{Posterior Mean}

To derive the posterior mean of $\mu_{p}$ given $X_{1}, \ldots, X_{p}$, we rely on the following classic result.

Lemma 1 (Normal Shrinkage). Let

$$
\mu \sim N\left(\mu_{0}, \nu^{2}\right), \quad Z_{i} \mid \mu \stackrel{i i d}{\sim} N\left(\mu, \tau^{2}\right) \quad(i=1, \ldots, n)
$$

Then the posterior mean of $\mu$ is

$$
\mathrm{E}\left(\mu \mid Z_{1}, \ldots, Z_{n}\right)=\frac{\tau^{2} \mu_{0}+\nu^{2} \sum_{i=1}^{n} Z_{i}}{\tau^{2}+n \nu^{2}}
$$

Proposition 1. The posterior mean of $\mu_{p}$ given $X_{p}$ is

$$
\mathrm{E}\left(\mu_{p} \mid X_{p}\right)=\frac{1}{1+\sigma^{2}} X_{p}
$$

Furthermore, let

$$
a=\gamma^{2}+\sigma^{2} \eta^{2}, \quad b=1-\gamma^{2}+\sigma^{2}\left(1-\eta^{2}\right)
$$

and

$$
r_{1}, \ldots, r_{p-1}=\frac{\sigma^{2}\left(\eta^{2}-\gamma^{2}\right)}{a(a+p b)}, \quad r_{p}=\frac{a+(p-1) b \gamma^{2}}{a(a+p b)}
$$

The posterior mean of $\mu_{p}$ given $X_{1}, \ldots, X_{p}$ is

$$
\mathrm{E}\left(\mu_{p} \mid X_{1}, \ldots X_{p}\right)=\sum_{i=1}^{p} r_{i} X_{i} .
$$

Proof of Proposition 1. To prove the first half, notice that

$$
\mu_{p} \sim N(0,1), \quad X_{p} \mid \mu_{p} \sim N\left(\mu_{p}, \sigma^{2}\right),
$$

and apply Lemma 1

To prove the second half, note that $\mu_{i}=\phi+\mu_{i}^{\prime}$, where

$$
\phi \sim N\left(0,1-\gamma^{2}\right), \quad \mu_{i}^{\prime} \stackrel{i i d}{\sim} N\left(0, \gamma^{2}\right)
$$


and $\epsilon_{i}=\xi+\epsilon_{i}^{\prime}$, where

$$
\xi \sim N\left\{0,\left(1-\eta^{2}\right) \sigma^{2}\right\}, \quad \epsilon_{i}^{\prime} \stackrel{i i d}{\sim} N\left(0, \eta^{2} \sigma^{2}\right)
$$

Consequently we have

$$
\phi+\xi \sim N(0, b), \quad X_{i} \mid \phi+\xi \stackrel{i i d}{\sim} N(\phi+\xi, a),
$$

On the one hand, by Lemma 1

$$
\mathrm{E}\left(\phi+\xi \mid X_{1}, \ldots X_{p}\right)=\frac{b}{a+p b} \sum_{i=1}^{p} X_{i}
$$

and

$$
\mathrm{E}\left(\phi \mid \phi+\xi, X_{1}, \ldots, X_{p}\right)=\frac{1-\gamma^{2}}{b} E\left(\phi+\xi \mid X_{1}, \ldots X_{p}\right) .
$$

Consequently,

$$
\begin{aligned}
\mathrm{E}\left(\phi \mid X_{1}, \ldots X_{p}\right) & =\mathrm{E}\left\{\mathrm{E}\left(\phi \mid \phi+\xi, X_{1}, \ldots, X_{p}\right) \mid X_{1}, \ldots X_{p}\right\} \\
& =\frac{1-\gamma^{2}}{b} E\left(\phi+\xi \mid X_{1}, \ldots X_{p}\right) \\
& =\frac{1-\gamma^{2}}{a+p b} \sum_{i=1}^{p} X_{i} .
\end{aligned}
$$

On the other hand, similarly we have

$$
\begin{aligned}
\mathrm{E}\left(\mu_{p}^{\prime} \mid X_{1}, \ldots X_{p}\right) & =\frac{\gamma^{2}}{a} E\left(\mu_{i}^{\prime}+\epsilon_{i}^{\prime} \mid X_{1}, \ldots X_{p}\right) \\
& =\frac{\gamma^{2}}{a}\left\{X_{p}-\frac{b}{a+p b} \sum_{i=1}^{p} X_{i}\right\}
\end{aligned}
$$

Combine (6) and (7), we complete the proof.

It is worth noting that when $\gamma=\eta$, (5) reduces to (4). 


\section{CLOSED-FORM EXPRESSION FOR THE SELECTION BIAS}

To simplify future notations, we assume that $X_{p}$ is the largest observation, i.e., $X_{p}=\max _{1 \leq i \leq p} X_{i}$. Consequently, the selection bias defined in (1) becomes

$$
\Delta=\mathrm{E}\left(\mu_{p} \mid X_{p}\right)-\mathrm{E}\left(\mu_{p} \mid X_{p}, X_{p}=\max X_{i}\right)
$$

To derive its closed-form expression, we rely on the following lemmas.

Lemma 2. Let $\boldsymbol{X}_{-p}=\left(X_{1}, \ldots, X_{p-1}\right)^{\prime}$, and its distribution conditioning on $X_{p}$ is

$$
N\left(\frac{b}{a+b} \mathbf{1}_{p-1} X_{p}, a \boldsymbol{I}_{p-1}+\frac{a b}{a+b} \mathbf{1}_{p-1} \mathbf{1}_{p-1}^{\prime}\right)
$$

Proof of Lemma 2. By (2) we have $\boldsymbol{X} \sim N(0, \boldsymbol{\Psi})$, where

$$
\boldsymbol{\Psi}=\left(\psi_{j k}\right)_{1 \leq j, k \leq p}=a \boldsymbol{I}_{p}+b \mathbf{1}_{p} \mathbf{1}_{p}^{\prime}
$$

Furthermore, let

$$
\boldsymbol{\Psi}_{11}=\left(\psi_{j k}\right)_{1 \leq j, k \leq p-1}=a \boldsymbol{I}_{p-1}+b \mathbf{1}_{p-1} \mathbf{1}_{p-1}^{\prime}, \quad \mathbf{\Psi}_{22}=\left(\psi_{p p}\right)=a+b
$$

and

$$
\boldsymbol{\Psi}_{12}=\left(\psi_{1 p}, \ldots, \psi_{p-1, p}\right)^{\prime}=b \mathbf{1}_{p-1}, \quad \boldsymbol{\Psi}_{21}=\left(\psi_{p 1}, \ldots, \psi_{p, p-1}\right)=b \mathbf{1}_{p-1}^{\prime}
$$

Simple probability argument suggests that

$$
\boldsymbol{X}_{-p} \mid X_{p} \sim N\left(\boldsymbol{\Psi}_{12}^{-1} \mathbf{\Psi}_{22} X_{p}, \boldsymbol{\Psi}_{11}-\boldsymbol{\Psi}_{12} \boldsymbol{\Psi}_{22}^{-1} \boldsymbol{\Psi}_{21}\right)
$$

where

$$
\mathbf{\Psi}_{12} \mathbf{\Psi}_{22}^{-1} X_{p}=\frac{b}{a+b} \mathbf{1}_{p-1} X_{p}
$$


and

$$
\begin{aligned}
\mathbf{\Psi}_{11}-\boldsymbol{\Psi}_{12} \boldsymbol{\Psi}_{22}^{-1} \boldsymbol{\Psi}_{21} & =a \boldsymbol{I}_{p-1}+b \mathbf{1}_{p-1} \mathbf{1}_{p-1}^{\prime}-\frac{b^{2}}{a+b} \mathbf{1}_{p-1} \mathbf{1}_{p-1}^{\prime} \\
& =a \boldsymbol{I}_{p-1}+\frac{a b}{a+b} \mathbf{1}_{p-1} \mathbf{1}_{p-1}^{\prime}
\end{aligned}
$$

The proof is complete.

To state the next lemma, we introduce some notations. First, for $\boldsymbol{\theta}=\left(\theta_{1}, \ldots, \theta_{n}\right)^{\prime}$ and positive semi-definite matrix $\boldsymbol{\Omega}=\left(\omega_{j k}\right)_{1 \leq j, k \leq n}$, let

$$
\boldsymbol{Y}=\left(Y_{1}, \ldots, Y_{n}\right)^{\prime} \sim N(\boldsymbol{\theta}, \boldsymbol{\Omega})
$$

Second, let $V_{i}=Y_{i}-\theta_{i}$ for $i=1, \ldots, n$. Consequently,

$$
\boldsymbol{V}=\left(V_{1}, \ldots, V_{n}\right)^{\prime} \sim N(\mathbf{0}, \boldsymbol{\Omega}),
$$

whose probability density function is

$$
f(\boldsymbol{v})=\frac{1}{(2 \pi)^{n / 2}|\boldsymbol{\Omega}|^{1 / 2}} \mathrm{e}^{-\frac{1}{2} \boldsymbol{v}^{\prime} \boldsymbol{\Omega}^{-1} \boldsymbol{v}}, \quad \boldsymbol{v}=\left(v_{1}, \ldots, v_{n}\right)^{\prime} .
$$

Third, for constants $b_{1}, \ldots, b_{n}$, we let

$$
\alpha=\operatorname{Pr}\left(V_{1} \leq b_{1}-\theta_{1}, \ldots, V_{n} \leq b_{n}-\theta_{n}\right)=\int_{v_{1} \leq b_{1}-\theta_{1}, \ldots, v_{n} \leq b_{n}-\theta_{n}} f(\boldsymbol{v}) d \boldsymbol{v}
$$

and $\boldsymbol{W}=\left(W_{1}, \ldots, W_{n}\right)^{\prime}$ be the truncation version of $\boldsymbol{V}$ from above at $\left(b_{1}-\theta_{1}, \ldots, b_{n}-\theta_{n}\right)^{\prime}$. Consequently, its probability density function is

$$
g(\boldsymbol{w})=\frac{1}{\alpha(2 \pi)^{n / 2}|\boldsymbol{\Omega}|^{1 / 2}} \mathrm{e}^{-\frac{1}{2} \boldsymbol{w}^{\prime} \boldsymbol{\Omega}^{-1} \boldsymbol{w}} \cdot 1_{\left\{w_{1} \leq b_{1}-\theta_{1}, \ldots, w_{n} \leq b_{n}-\theta_{n}\right\}}, \quad \boldsymbol{w}=\left(w_{1}, \ldots, w_{n}\right)^{\prime}
$$


For all $k=1, \ldots, n$, let the $k$ th marginal density function of $\boldsymbol{W}$ be

$$
g_{k}(w)=\int_{-\infty}^{b_{1}-\theta_{1}} \cdots \int_{-\infty}^{b_{k-1}-\theta_{k-1}} \int_{-\infty}^{b_{k+1}-\theta_{k+1}} \ldots \int_{-\infty}^{b_{n}-\theta_{n}} g\left(w_{1}, \ldots, w_{k-1}, w, w_{k+1}, \ldots, w_{n}\right) \prod_{l \neq k} d w_{l} .
$$

For efficient analytical and numerical evaluations of (10), see Cartinhour (1990) and Wilhelm and Manjunath (2010), respectively.

Lemma 3. For all $i=1, \ldots, n$,

$$
\mathrm{E}\left(Y_{i} \mid Y_{1} \leq b_{1}, \ldots, Y_{n} \leq b_{n}\right)=\theta_{i}-\sum_{k=1}^{n} \omega_{k i} g_{k}\left(b_{k}-\theta_{k}\right)
$$

Proof of Lemma 3. The proof follows Manjunath and Wilhelm (2012). First,

$$
\begin{aligned}
\mathrm{E}\left(Y_{i} \mid Y_{1} \leq b_{1}, \ldots, Y_{n} \leq b_{n}\right) & =\theta_{i}+E\left(V_{i} \mid V_{1} \leq b_{1}-\theta_{1}, \ldots, V_{n} \leq b_{n}-\theta_{n}\right) \\
& =\theta_{i}+E\left(W_{i}\right) .
\end{aligned}
$$

Next, the moment generating function of $\boldsymbol{W}$ at $\boldsymbol{t}=\left(t_{1}, \ldots, t_{n}\right)^{\prime}$ is

$$
\begin{aligned}
m(\boldsymbol{t}) & =\int \mathrm{e}^{\boldsymbol{t}^{\prime} \boldsymbol{w}} g(\boldsymbol{w}) d \boldsymbol{w} \\
& =\frac{1}{\alpha(2 \pi)^{n / 2}|\boldsymbol{\Omega}|^{1 / 2}} \int_{w_{1} \leq b_{1}-\theta_{1}, \ldots, w_{n} \leq b_{n}-\theta_{n}} \mathrm{e}^{-\frac{1}{2}\left(\boldsymbol{w}^{\prime} \boldsymbol{\Omega}^{-1} \boldsymbol{w}-2 \boldsymbol{t}^{\prime} \boldsymbol{w}\right)} d \boldsymbol{w} \\
& =\underbrace{\mathrm{e}^{\frac{1}{2} \boldsymbol{t}^{\prime} \boldsymbol{\Omega}}}_{m_{1}(\boldsymbol{t})} \underbrace{\frac{1}{\alpha(2 \pi)^{n / 2}|\boldsymbol{\Omega}|^{1 / 2}} \int_{w_{1} \leq b_{1}-\theta_{1}, \ldots, w_{n} \leq b_{n}-\theta_{n}} \mathrm{e}^{-\frac{1}{2}(\boldsymbol{w}-\boldsymbol{\Omega} \boldsymbol{t})^{\prime} \boldsymbol{\Omega}^{-1}(\boldsymbol{w}-\boldsymbol{\Omega} \boldsymbol{t})} d \boldsymbol{w}}_{m_{2}(\boldsymbol{t})}
\end{aligned}
$$

On the one hand, by definition

$$
\begin{aligned}
E\left(W_{i}\right) & =\left.\frac{\partial m(\boldsymbol{t})}{\partial t_{i}}\right|_{\boldsymbol{t}=\mathbf{0}} \\
& =\left.m_{1}(\mathbf{0}) \frac{\partial m_{2}(\boldsymbol{t})}{\partial t_{i}}\right|_{\boldsymbol{t}=\mathbf{0}}+\left.m_{2}(\mathbf{0}) \frac{\partial m_{1}(\boldsymbol{t})}{\partial t_{i}}\right|_{\boldsymbol{t}=\mathbf{0}} \\
& =\left.\frac{\partial m_{2}(\boldsymbol{t})}{\partial t_{i}}\right|_{\boldsymbol{t}=\mathbf{0}} .
\end{aligned}
$$


On the other hand, let

$$
b_{i}^{*}=b_{i}-\theta_{i}-\sum_{k=1}^{n} \omega_{i k} t_{k}, \quad i=1, \ldots, n,
$$

and we can rewrite $m_{2}(\boldsymbol{t})$ as

$$
m_{2}(\boldsymbol{t})=\int_{-\infty}^{b_{1}^{*}} \ldots \int_{-\infty}^{b_{n}^{*}} g(\boldsymbol{w}) d w_{1} \ldots d w_{n}
$$

Therefore, by chain rule and Leibniz integral rule

$$
\begin{aligned}
\frac{\partial m_{2}(\boldsymbol{t})}{\partial t_{i}} & =\sum_{k=1}^{n} \frac{\partial b_{k}^{*}}{\partial t_{i}} \frac{\partial m_{2}(\boldsymbol{t})}{\partial b_{k}^{*}} \\
& =-\sum_{k=1}^{n} \omega_{k i} \int_{-\infty}^{b_{1}^{*}} \ldots \int_{-\infty}^{b_{k-1}^{*}} \int_{-\infty}^{b_{k+1}^{*}} \cdots \int_{-\infty}^{b_{n}^{*}} g\left(w_{1}, \ldots, w_{k-1}, b_{k}^{*}, w_{k+1}, \ldots, w_{n}\right) \prod_{l \neq k} d w_{l},
\end{aligned}
$$

and consequently

$$
\left.\frac{\partial m_{2}(\boldsymbol{t})}{\partial t_{i}}\right|_{\boldsymbol{t}=0}=-\sum_{k=1}^{n} \omega_{k i} g_{k}\left(b_{k}-\theta_{k}\right) .
$$

Combine (11), 12 and (13), the proof is complete.

Proposition 2. For $i=1, \ldots, p-1$, let $h_{i}$ denote the $i$ th marginal probability density function of the random vector defined by (9) truncated from above at $\mathbf{1}_{p-1} X_{p}$. Then the closed-form expression for $(8)$ is

$$
\Delta=\frac{\sigma^{2}\left(\eta^{2}-\gamma^{2}\right)}{1+\sigma^{2}} \sum_{i=1}^{p-1} h_{i}\left(\frac{\gamma^{2}+\sigma^{2} \eta^{2}}{1+\sigma^{2}} X_{p}\right) .
$$

Proof of Proposition 2. Apply Lemma 2 and 3 to (9),

$$
E\left(X_{i} \mid X_{p}, X_{p}=\max X_{i}\right)=\frac{a}{a+b} X_{p}-\underbrace{\left\{\frac{a b}{a+b} \sum_{j=1}^{p-1} h_{j}\left(\frac{a}{a+b} X_{p}\right)+a h_{i}\left(\frac{a}{a+b} X_{p}\right)\right\}}_{\delta_{i}} .
$$


Consequently, by (5) we have

$$
\begin{aligned}
\mathrm{E}\left(\mu_{p} \mid X_{p}, X_{p}=\max X_{i}\right) & =r_{p} X_{p}+\sum_{i=1}^{p-1} r_{i} \mathrm{E}\left(X_{i} \mid X_{p}, X_{p}=\max X_{i}\right) \\
& =\left(r_{p}+\frac{a}{a+b} \sum_{i=1}^{p-1} r_{i}\right) X_{p}-\sum_{i=1}^{p-1} r_{i} \delta_{i} \\
& =\frac{X_{p}}{a+b}+\left\{\frac{(p-1) a b}{a+b}+a\right\} \sum_{i=1}^{p-1} r_{i} h_{i}\left(\frac{a}{a+b} X_{p}\right) \\
& =\mathrm{E}\left(\mu_{p} \mid X_{p}\right)-\frac{\sigma^{2}\left(\eta^{2}-\gamma^{2}\right)}{1+\sigma^{2}} \sum_{i=1}^{p-1} h_{i}\left(\frac{\gamma^{2}+\sigma^{2} \eta^{2}}{1+\sigma^{2}} X_{p}\right)
\end{aligned}
$$

The proof is complete.

Proposition 2 confirms the existence of the selection bias in general. Furthermore, it provides the following interesting insights:

1. For fixed $\sigma, p$ and $X_{p}$, the sign of the selection bias is the same as the sign of $\eta^{2}-\gamma^{2}$, i.e., depending on the correlation structures in (3), neglecting the fact that $X_{p}=\max _{1 \leq i \leq p} X_{i}$ can either over-estimate or under-estimate $\mu_{i^{*}}$. In particular, the selection bias is zero when $\gamma=\eta$. This is a generalization of the first main result in Senn (2008), which assumes that $\gamma=\eta=1$

2. For fixed $\gamma, \eta, p$ and $X_{p}$, the selection bias goes to zero as $\sigma$ goes to zero. This is intuitive because $X_{p}$ approaches $\mu_{p}$ as $\sigma$ goes to zero, and therefore the fact that $X_{p}=\max _{1 \leq i \leq p} X_{i}$ becomes irrelevant;

3. For fixed $\sigma, \gamma, \eta$ and $p$, the selection bias disappears for sufficiently large $X_{p}$. This is because when $X_{p}$ goes to infinity,

$$
h_{i}\left(\frac{\sigma^{2}+\gamma^{2} \eta^{2}}{1+\sigma^{2}} X_{p}\right) \rightarrow 0, \quad i=1, \ldots, p-1
$$

This result is in connection with Dawid (1973). 


\section{NUMERICAL AND SIMULATED EXAMPLES}

\subsection{Numerical Examples}

Having derived the closed-form expression for the selection bias, we provide some numerical examples for illustration. Let $\sigma=1, p \in\{3,5,10\}$ and $X_{p} \in\{0,1, \ldots, 6\}$. For fixed $p$ and $X_{p}$, we consider two cases. In Case 1, we follow $\operatorname{Senn}(2008)$ and let $\gamma^{2}=0.5$ and $\eta=1$. In Case 2, we let $\gamma=1$ and $\eta^{2}=0.5$. For both cases we calculate the selection bias by (14). Results are in Figure 1 . which align with the insights discussed in the previous section. Furthermore, it appears that the magnitude of the selection bias increases as $p$ increases.
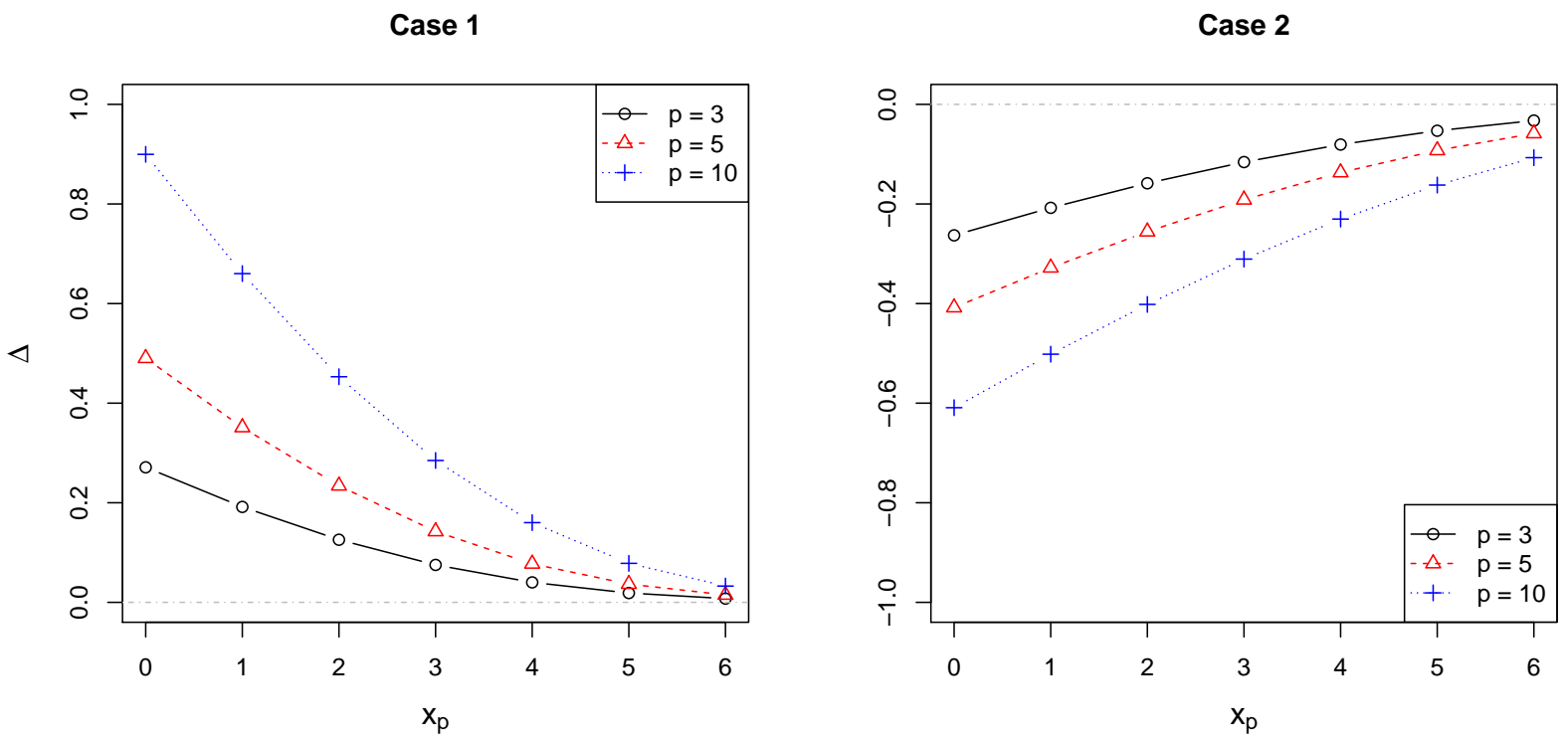

Figure 1: Numerical Examples of Selection Bias.

\subsection{Simulated Examples}

The results in (14) enable us to calculate the "exact post-selection" posterior mean

$$
\lambda_{i^{*}}=\mathrm{E}\left(\mu_{i^{*}} \mid X_{i^{*}}, X_{i^{*}}=\max X_{i}\right)
$$

For illustration, we revisit the simulated example in $\operatorname{Senn}(2008)$, where $p=10, \sigma=2, \gamma^{2}=0.5$ and $\eta=1$. Figure 2 contains 5000 pairs of $\left(\mu_{i^{*}}, X_{i^{*}}\right)$ obtained by repeated sampling, the corresponding 
linear regression line that Senn (2008) used to approximate (15), and the curve that stands for the closed-form expression for (15).

The results in Figure 2 suggest that the regression approximation is relatively accurate for nonextreme values of $X_{i^{*}}$ but not for extreme ones. Therefore our analytical solution has an advantage over the regression approximation in Senn (2008). For further illustration we examine two concrete examples. First, let

$$
x_{i^{*}}=3.25, \quad \operatorname{Pr}\left(X_{i^{*}}>x_{i^{*}}\right)=0.486 .
$$

Therefore 3.25 is a "common" value of $X_{i^{*}}$. In this case the exact value of 115 is $\lambda_{i^{*}}=0.400$ and the regression approximation is $\hat{\lambda}_{i^{*}}=0.368$. Consequently, although the "absolute discrepancy" $\left|\hat{\lambda}_{i^{*}}-\lambda_{i^{*}}\right|=0.032$ seems small, the "relative discrepancy"

$$
\frac{\left|\hat{\lambda}_{i^{*}}-\lambda_{i^{*}}\right|}{\left|\lambda_{i^{*}}\right|}=8.1 \%
$$

is moderately large. Second, let

$$
x_{i^{*}}=1.5, \quad \operatorname{Pr}\left(X_{i^{*}} \leq x_{i^{*}}\right)=0.102 .
$$

Therefore 1.5 is a relatively "uncommon" (but not extreme) value of $X_{i^{*}}$. In this case the absolute and relative discrepancies are respectively 0.062 and $24.7 \%$, both moderately large.

\section{CONCLUDING REMARKS}

For the treatment selection problem, quantifying the selection bias is important from both theoretical and practical perspectives. In this paper, we extend the heuristic discussion in Senn (2008) and derive the closed-form expression for the selection bias. We illustrate the advantages of our results by numerical and simulated examples.

There are multiple possible future directions based on our current work. First, we can reconcile our Bayesian analysis with Frequentist methods. Second, it is possible to extend our results to more general model specifications by using the Tweedie's formula (Robbins 1956; Efron 2011). Third, we need to explore "exact post-selection inference" in multiple hypothesis testing. 


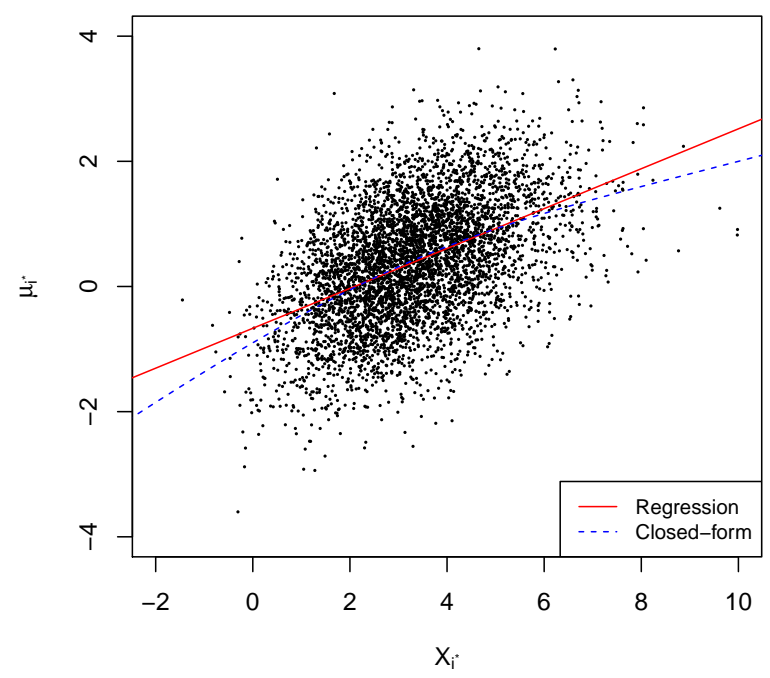

Figure 2: "Exact Post-Selection" Posterior Mean: Regression Approximation (Red Solid Line) and Closed-Form Expression (Blue Dotted Line).

\section{ACKNOWLEDGEMENTS}

The authors thank the Co-Editor-in-Chief and a reviewer for their thoughtful comments that substantially improved the presentation of the paper.

\section{REFERENCES}

Cartinhour, J. (1990). One-dimensional marginal density functions of a truncated multivariate normal density function. Communication in Statistics - Theorey and Methods, 17(1):197-203.

Dawid, A. P. (1973). Posterior expectations for large observations. Biometrika, 60(3):664-667.

Dawid, A. P. (1994). Selection paradoxes of Bayesian inference. Mvdtiaate Analysis and Its Applications, Lecture Notes - Monograph Series, 24:211-220.

Efron, B. (2011). Tweedie's formula and selection bias. Journal of the American Statistical Association, 106(496):1602-1614.

G'Sell, M. G., Wager, S., Chouldechova, A., and Tibshirani, R. (2016). Sequential selection pro- 
cedures and false discovery rate control. Journal of the Royal Statistical Society: Series B (Statistical Methodology), 78(2):423-444.

Kohavi, R., Deng, A., Frasca, B., Walker, T., Xu, Y., and Pohlmann, N. (2013). Online controlled experiments at large scale. In Proceedings of the 19th ACM SIGKDD international conference on Knowledge discovery and data mining, pages 1168-1176.

Lee, J. D., Sun, D. L., Sun, Y., and Taylor, J. (2015). Exact post-selection inference, with applications to the Lasso. Annals of Statistics, in press.

Lockhart, R., Taylor, J., Tibshirani, R. J., and Tibshirani, R. (2014). A significance test for the Lasso. Annals of Statistics, 42(2):413-468.

Manjunath, B. G. and Wilhelm, S. (2012). Moments calculation for the doubly truncated multivariate normal density. arXiv:1206.538\%.

Reid, S. and Tibshirani, R. (2016). Sparse regression and marginal testing using cluster prototypes. Biostatistics, 17(2):364-376.

Robbins, H. (1956). An empirical bayes approach to statistics. In Proceedings of the Third Berkeley Symposium on Mathematical Statistics and Probability, volume I, pages 157-163. University of California Press, Berkeley and Los Angeles.

Senn, S. (2008). A note concerning a selection "paradox" of Dawid's. The American Statistician, $62(3): 206-210$.

Taylor, J. and Tibshirani, R. (2015). Statistical learning and selective inference. Proceedings of the National Academy of Sciences, 112(25):7629-7634.

Tibshirani, R. J., Taylor, J., Lockhart, R., and Tibshirani, R. (2014). Exact post-selection inference for sequential regression procedures. Journal of the American Statistical Association, in press.

Wilhelm, S. and Manjunath, B. G. (2010). tmvtnorm: A package for the truncated multivariate normal distribution. The $R$ Journal, 2(1):25-29. 